\title{
Dried Extracts and Essential Oils of Some Pelargonium Species: Chemical and Biological Assessment
}

\author{
Cristina lancu, Oana Cioanca, Monica Hancianu, Cornelia Mircea
}

Grigore T. Popa" University of Medicine and Pharmacy, lasi, ROMANIA.

\begin{abstract}
Our aim was to evaluate the beneficial properties of the most important groups of compounds present in the leaves of Pelargonium hispidum, $\mathrm{P}$ grandiflorum and $\mathrm{P}$. radens. Given the studied species have a high content of polyphenols, the biologic activity was assessed by determination of free radical scavenging capacity against DPPH, and the investigation of superoxide anion radical scavenging capacity. The volatile fractions from the Pelargonium samples were separated by distillation with water vapors. Conditions such as the biological culture, temperature, humidity and natural lighting, influence plant production of volatile components. For the investigated samples, which grew under the same conditions, the species that had the highest quantity of essential oil was $P$. hispidum. Gas-chromatographic analysis of volatile oils from Pelargonium: hispidum, $P$. grandiflorum and $P$. radens revealed significant differences in the number and the type of volatile components. These variations were not surprising, given the different macroscopic and microscopic characteristics; yet, the low extraction yield did not allow the detailed biological study of these volatile compounds.
\end{abstract}

Key words: Pelargonium, Essential Oils, Gas-Chromatography, Antioxidant Activity, polyphenols.

\section{INTRODUCTION}

History proves that herbs are preferred as remedies regardless of the social and industrial level of development. Although there have been periods when different synthetic drugs were considered irreplaceable and eclipsed plant sources, once with the world development and the appearance of many illnesses of the modern civilization, the scientific world once again turned its attention to herbs, hoping to find valuable solutions for modern therapy. ${ }^{1}$ Thus, there are several premises that require research regarding the exploitation of natural resources with obvious therapeutic value. Therefore, our attention focused on easily accessible species on which there is little information in the literature from a chemical and biological point of view, and we started from the information available in the traditional medicine. In this sense, the purpose of this paper is to highlight new sources related to this species of Pelargonium (as this one is less accessible in our areas), which would constitute important therapeutic resources. Thus, the present study tries to highlight several biological and chemical data on the species acclimatized in Romania, while making correlations with data in the literature.

\section{MATERIAL AND METHODS}

\section{Plant material and extraction of compounds}

All samples (dried mature leaves collected at the same time from plants grown in similar conditions) were processed following the same procedure: $2 \mathrm{~g}$ of plant product were extracted with ethanol $50^{\circ}$ and absolute methanol for 45 minutes. After cooling, the extracts were transferred into capsules of sizes suitable for concentration in the $40^{\circ} \mathrm{C}$ thermostatic oven. The samples were coded as: P1 (P. hispidum), P2 (P. grandiflorum), P3 (P. radens). Depending on the
DOI: 10.5530/ijper.51.3s.60 Correspondence:

Cioancă Oana,

Grigore T. Popa" University of Medicine and Pharmacy, Iași, ROMANIA

Contact: +40232301816

E-Mail: oanacioanca@gmail. com 
type of extract, the corresponding index was added to the code name: $\mathrm{E}$ (hydro-alcoholic) or $\mathrm{M}$ (methanol), depending on the extraction solvent used. The volatile fractions from the Pelargonium samples under analysis were separated using the method of separation by distillation with water vapors. ${ }^{2}$

\section{The chemical profile assessment}

The total phenol content of the samples was quantified using the Folin-Ciocâlteu reagent, polyphenols yielded blue compounds for $\lambda=765 \mathrm{~nm}$. The general chemical profile of the volatile fractions was established by GC-MS-FID method.

\section{The investigation of the biologic activity \\ Determination of free-radical scavenging capacity (radical DPPH)}

When the solution is treated with a free radical scavenger, what results is the diphenyl-picrylhydrazyl (DPPH-H), with a change in color from purple to yellow and with a decrease in the maximum absorbance at $517 \mathrm{~nm}$.

\section{Superoxide anion radical scavenging capacity}

The superoxide anion radical, generated by the nicotinamide-adenine-dinucleotide (reduced)-phenazine methosulphate reduces tetrazolium nitrate (NBT) to a blue formazan with absorbance at $560 \mathrm{~nm}$.

\section{RESULTS AND DISCUSSIONS}

\section{Extractability}

The values recorded for the drug-extract ratio (DER) of the hydro-alcoholic extracts and the methanolic extracts are given in Table 1.

The results obtained by plant product mass calculation showed that the best extraction solvent was $50^{c}$ ethanol. From the point of view of the extractive capacity with both types of solvents, the best plant product proved to be the one of the Pelargonium radens species. At the opposite end was the plant product (the leaves) of the

Table 1: The amount of extract obtained from
Pelargonium samples
\begin{tabular}{|c|c|c|c|c|c|}
\multirow{2}{*}{ Code } & \multirow{2}{*}{ Source } & \multicolumn{2}{|c|}{ Extractible } & \multicolumn{2}{c|}{ DER $^{*}$ m/m } \\
\cline { 3 - 6 } & & E $^{* *}$ & M $^{* *}$ & E $^{* *}$ & M $^{* *}$ \\
\hline P1 & P. hispidum & $0,58837 \mathrm{~g}$ & $0,3155 \mathrm{~g}$ & 3,3992 & 6,3391 \\
\hline P2 & P. grandiflorum & $0,59366 \mathrm{~g}$ & $0,2745 \mathrm{~g}$ & 3,3689 & 7,2859 \\
\hline P3 & P. radens & $0,48753 \mathrm{~g}$ & $0,2546 \mathrm{~g}$ & 4,1023 & 7,8554 \\
\hline
\end{tabular}

*DER = plant product mass: obtained extract mass; ** E- hydro-alcoholic extract, $* * * M-$ methanol extract
Pelargonium hispidum species, which yielded the lowest quantities of extracts.

\section{Quantitative determination of the total polyphenols}

Particularly for our extracts, we proved that polar solvents favored a better extractability; thus, in the pharmaceutical practice, the solubility of the plant material compounds must be considered. The quantitative determination of flavonoids for ethanol extracts categorizes the species by ascending figures, as follows: $\mathrm{P} 3<\mathrm{P} 1<$ $\mathrm{P} 2$, compared to methanol extracts whose quantitative evolution is: $\mathrm{P} 2<\mathrm{P} 3<\mathrm{P} 1$.

\section{Volatile fractions}

For the investigated samples, which grew under the same conditions, the specie that had the highest quantity of essential oil was P. hispidum $(1,167 \%)$ compared to $P$. grandiflorum $(0,667 \%)$ and $P$. radens $(0,834 \%)$.

The gas-chromatographic analysis of the volatile oils revealed significant differences in the number and the type of the identified volatile components. ${ }^{3}$ These variations were not surprising, given the different macroscopic and microscopic characteristics of each species; yet, the low extraction yield did not allow the detailed biological study of these volatile compounds. The GC-MS and GC-FID profile of the chromatograms recorded for the volatile oils are shown in Figure 1.

In the case of Pelargonium grandiflorum volatile oil, compounds such as linalool, geraniol derivatives and cis-rose oxide were detected, compared to P. hispidum for which the most important compounds were menthone (15.70\%), thymol, pinene and eucalyptol.

On the other hand, the major volatile component of P. radens leaves was isomenthone (85\%), accompanied by very small quantities of pinene, terpinene, limonene, myrcene, cymene and piperitone.

\section{The biologic activity}

\section{Determination of free-radical scavenging capacity (radical DPPH)}

DPPH radical is a synthetic compound which is not found in the living world, but which is frequently used for the screening of the antioxidant potential due to its increased reactivity to natural compounds. ${ }^{4,5}$ If we plot all six extracts investigated on a downward axis showing their antioxidant potential, we will get the following scale: P1M $>$ P2E $>$ P3M $>$ P1E $>$ P2M $>$ P3E.

\section{Superoxide anion radical scavenging capacity}

It is widely accepted that oxidative stress causes damage at the cellular level by accelerating cellular aging mechanisms extrapolated to the entire body. ${ }^{6,7}$ Therefore, the active principles which have antioxidant effects have 

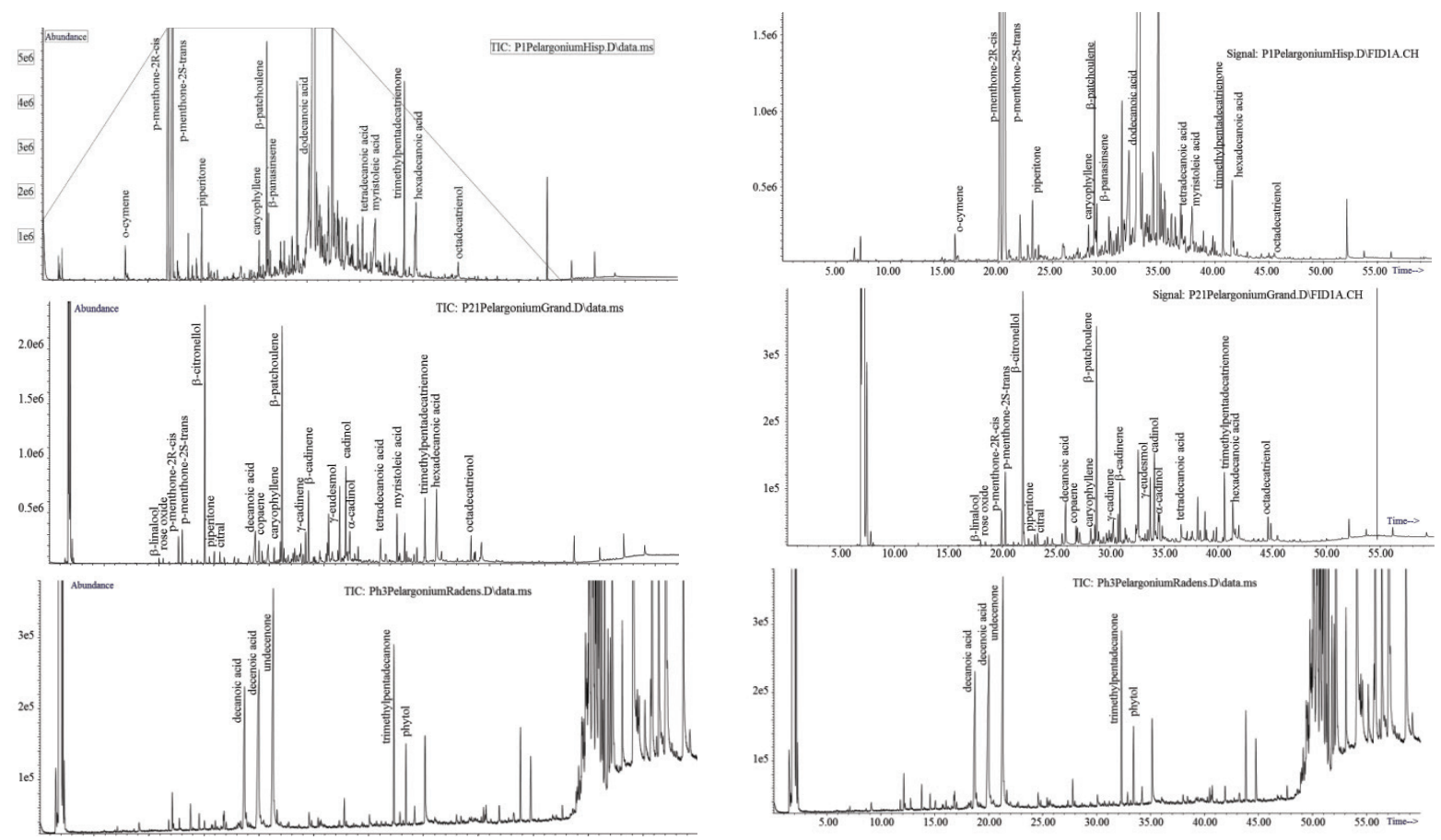

Figure1: GC-MS profile (left) and GC-FID chromatograms (right) of the oils extracted from the analyzed species: P. hispidum, $P$. grandiflorum, $P$. radens

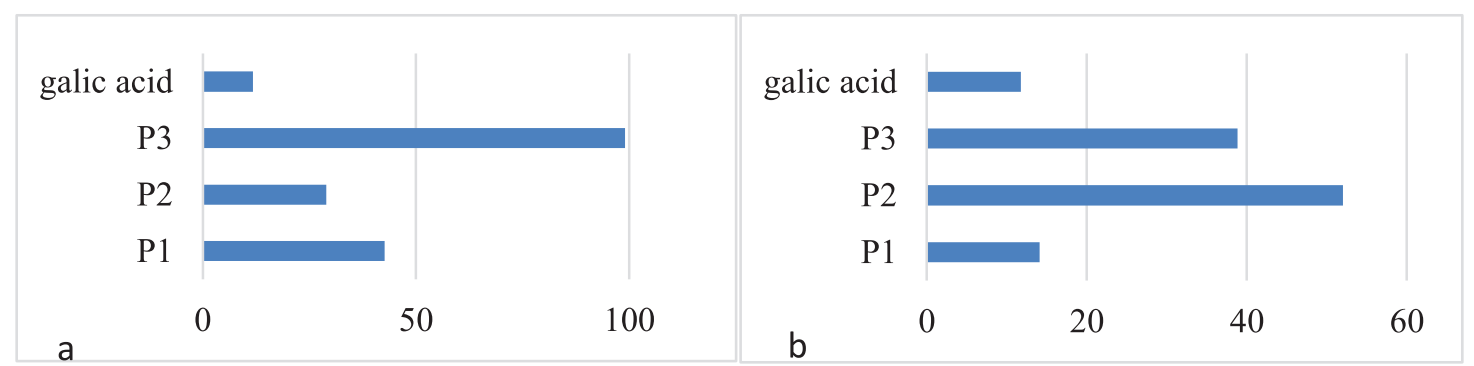

Figure 2: Graphical representation of the EC50 values $(\mu \mathrm{g} / \mathrm{mL})$ obtained in the evaluation of ethanol extracts (a) and methanol extracts (b) with the DPPH test

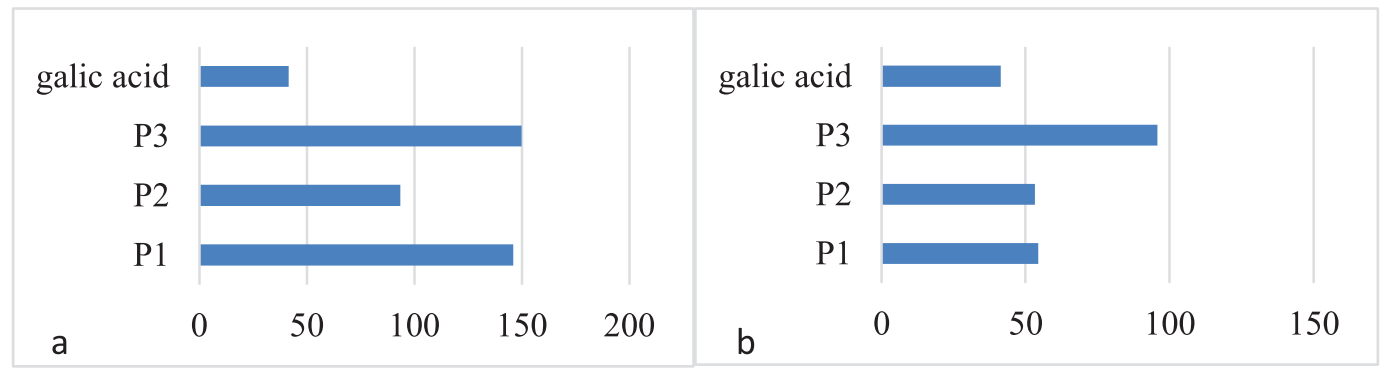

Figure 3: Graphical representation of EC50 $(\mu \mathrm{g} / \mathrm{mL})$ of ethanol extracts (a) and methanol extracts (b) in the superoxide anion radical scavenging capacity test

increasingly caught researchers' interest in recent years. ${ }^{8}$ Such results indicate that the type of the solvent is extremely important for the proper extractability of the most active compounds, some of which might be stronger scavengers than the others. The descending order of the superoxide anion radical scavenging capacity was:
$\mathrm{P} 2 \mathrm{M}>\mathrm{P} 1 \mathrm{M}>\mathrm{P} 2 \mathrm{E}>\mathrm{P} 3 \mathrm{M}>\mathrm{P} 1 \mathrm{E}>\mathrm{P} 3 \mathrm{E}$. The compounds responsible for the antioxidant activity of plant extracts are, in general, the total polyphenols. However, the results show that not necessarily the same extracts are the best choice for both type of antioxidant assays. 


\section{CONCLUSION}

The investigated species P. hispidum, P. grandiflorum, $P$. radens presented interspecific variations in regard to their volatile and phenolic compounds. The methanolic extracts showed a better antioxidant potential, therefore ongoing studies try to evaluate the true potential of the compounds isolated from Pelargonium species grown in Romania.

\section{ACKNOWLEDGEMENT}

Nil

\section{CONFLICT OF INTEREST}

The authors declare that there is no conflict of interest regarding the results included in this paper.

\section{ABBREVIATION USED}

DPPH: 2,2 diphenyl-1-picrylhydrazyl; P1: Pelargonium hispidum; P2: Pelargonium grandiflorum; P3: Pelargonium radens; E: hydro-alcoholic extract; $\mathrm{M}$ : methanolic

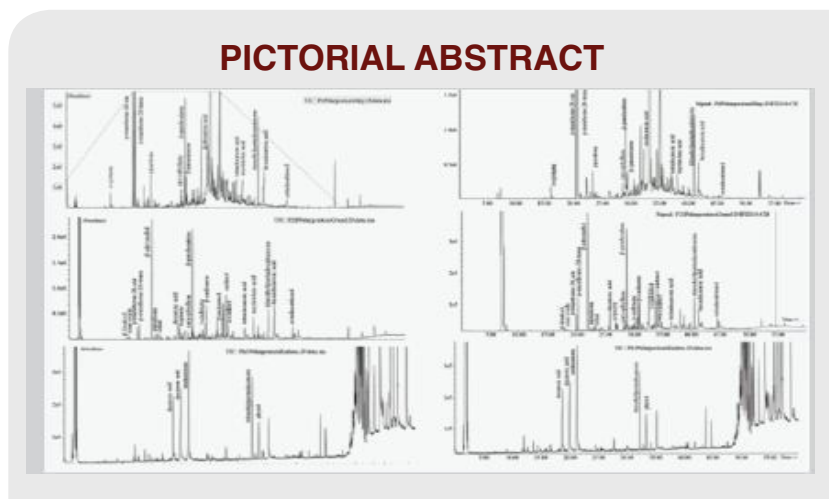

\section{ABOUT AUTHORS}

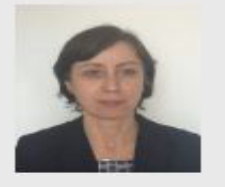

Mircea Cornelia: Assoc prof. Department of Biochemistry and Clinical Laboratory, at "Grigore T. Popa" University of Medicine and Pharmacy lasi;; expertise in enzymatic approaches of both natural and synthetic compounds

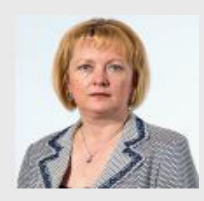

Hancianu Monica: Professor in Department of Pharmacognosy and Phytotherapy, at "Grigore T. Popa" University of Medicine and Pharmacy lasi; expertize in the assessment and quality control of medicinal plants, herbal extracts, herbal active compounds and herbal drugs preparations extract; GC-MS-FID: gas chromatography-mass spectrometer-flame ion detector; NBT: tetrazolium nitrate; DER: drug-extract ratio $\mathrm{EC}_{50}$ : half maximal effective concentration

\section{REFERENCES}

1. Bladt S. Zur Chemie der Inhaltsstoffe der Pelargonium reniforme CURT. - Wurzel (Umckaloabo). München: Dissertation, Ludwig-MaximiliansUniversität, 1974.

2. ${ }^{* * *}$ Farmacopeea Română, ediția a X-a. Bucureşti: Editura Medicală, 1993.

3. Gacea O. Cercetări privind calitatea farmaceutică a produsului Chamomillae flos. UMF, Gr.T.Popa” Iași, 2010.

4. Cioanca O, Mircea C, Hritcu L, Trifan A, Mihasan M, Aprotosoaie AC, et al. In vitro-in vivo correlation of the antioxidant capacity of Salvie aetheroleum essential oil. Farmacia. 2015:63(1):34-9.

5. Malterud KE, Farbrot TL, Huse A., Sund RB. Antioxidant and radicalscavenging effects of anthraquinones and anthrones. Pharmacol. 1993; $47: 77-85$

6. Prior RL, Wu XL, Schaich K. Standardized methods for the determination of antioxidant capacity and phenolics in foods and dietary supplements. J Agric Food Chem. 2005;53(10):4290-302.

7. Csepregi $\mathrm{K}$, Kocsis M, Hideg É. On the spectrophotometric determination of total phenolic and flavonoid contents. Acta Biol Hung. 2013;64:509-18.

8. Harman D. origin and evaluation of the free radical theory of aging: a brief personal history. Biogerontology. 2009;10(6):773-81.

\section{SUMMARY}

- The most important groups of compounds from the leaves of Pelargonium hispidum, $P$ grandiflorum and $P$. radens were investigated

- Polar solvents favored a better extractability of the phenolic compounds

- $P$. radens essential oil had high content of isomenthone

- Linalool, geraniol derivatives and cis-rose oxide were detected in Pelargonium grandiflorum essential oil.

- The most important compounds in P. hispidum essential oil were menthone $(15.70 \%)$, thymol, pinene and eucalyptol.

- The downward axis showing the scavenger potential against DPPH radical for the investigated extracts is: $\mathrm{P} 1 \mathrm{M}>\mathrm{P} 2 \mathrm{E}>\mathrm{P} 3 \mathrm{M}>\mathrm{P} 1 \mathrm{E}>\mathrm{P} 2 \mathrm{M}>\mathrm{P} 3 \mathrm{E}$

- The descending order of the superoxide anion radical scavenging capacity was: P2M $>$ P1M $>$ P2E $>$ P3M > P1E > P3E.

- The methanolic extracts showed a better antioxidant potential than the other type of extracts.

Cite this article: lancu C, Cioanca O, Hancianu M, Mircea C. Dried Extracts and Essetial Oils of Some Pelargonium Species: Chemical and Biological Assesment. Indian J of Pharmaceutical Education and Research. 2017;51(3) Suppl:S421-24. 